\title{
A tención y tiempo de reacción en practicantes de kárate Shotokan
}

António Vences de Brito, Carlos Silva, luis Cid, Dora Ferreira, Ana Marques

IPS - Escola Superior de Desporto de Rio Maior (Portugal).

Recibido 07/10/2010 - Aceptado 03/02/2011

\section{Resumen}

El objetivo del presente estudio es analizar la capacidad de atención y el tiempo de reacción en practicantes de kárate portugueses. Participaron 96 karatecas Shotokan federados, pertenecientes al Centro Português de Karate, de los que se recogieron medidas antropométricas (peso, altura, índice de masa corporal y porcentaje de masa grasa), y fueron evaluados en cuanto al Tiempo de Reacción Simple (TRS), Tiempo de Reacción de Elección (TRE), Tiempo de Decisión (TD) y Atención Distribuida (AD). Los datos fueron analizados de acuerdo a la edad de los karatecas (15-19, 20-35 y +35 años), graduación (90-40 kyu, $3^{\text {er- }} 1^{\text {er }} \mathrm{kyu}$, dan) y género (masculino y femenino). Los resultados muestran que los karatecas masculinos presentan características físicas significativamente diferentes en cuanto a altura, peso, porcentaje de masa grasa y años de práctica respecto a las practicantes femeninas. En cuanto al TRS todos los grupos tienden a que sus respuestas ocurran 300 ms después del estímulo, sin existir diferencias significativas entre ellos. En relación al TRE y TD, los karatecas con graduación de dan y más de 35 años presentan tiempos peores que los restantes grupos. Por otro lado, los karatecas con mayor graduación (dan) y más de 35 años tienden a cometer menos errores en sus elecciones. La variable género no influye significativamente al TR de los practicantes, aunque parece que las mujeres evaluadas tienden a reaccionar más rápidamente que los hombres. En cuanto a la $A D$, podemos concluir que no existen diferencias significativas en función de la graduación del atleta ni en función del género. Encontramos diferencias estadísticamente significativas en función de la edad, los atletas mayores fueron aquellos que presentan niveles inferiores de atención distribuida. Todo ello parece indicar que son necesarias modificaciones en el proceso de entrenamiento de los karatecas Shotokan en Portugal.

Palabras clave: Atención, tiempo de reacción, procesamiento de la información, kárate, rendimiento.

Attention and Reaction Time in Shotokan Athletes

Abstract: The aim of this study is to analyze the attention capacity and the reaction time in Portuguese karate Shotokan athletes. Participated 96 Shotokan athletes from the 
Portuguese Karate Association. We physically characterized the sample (weight, height, body mass index, and body fat mass percentage) and evaluated Simple Reaction Time (TRS), Choice Reaction Time (TRE), Decision Time (TD) and the Distributed Attention (AD). Data was analyzed according to athletes' group age (15 to $19 \mathrm{yr}, 20$ to $35 \mathrm{yr}$ and more than $35 \mathrm{yr}$ ), level of graduation ( $9^{\text {th }}$ to $4^{\text {th }} \mathrm{kyu}, 3^{\text {rd }}$ to $1^{\text {st }} \mathrm{kyu}, \mathrm{DAN}$ ) and by gender (male and female). Male athletes present significant differences from female athletes in height, weight, years of practice and body fat mass. In relation to TRS all groups tend to a value near to 300 ms without significant differences among them, but the TRE and the TD are significantly higher in the Dan athletes and in the +35 yrs athletes than in the other groups. On the other hand the Dan and +35 yrs athletes tend to do less mistakes. Gender does not influence significantly the reaction time in the Shotokan karate athletes, but it seems that women tend to have smaller reaction times than men. Athletes with more years of practice and more graduation need more time to reply to the stimulus than the other athletes, but they tend to do fewer mistakes on their choices than other subjects. As for distributed attention, no significant differences were found in function of the athlete graduation, nor in function of gender. However, for distributed attention, we found statistical significant differences in function of the age, with the oldest athletes presenting lower levels of distributed attention. Our results seem to show that is necessary to do some modifications in the training process of Portuguese Shotokan karate athletes.

Key words: Attention, reaction time, information processing, karate, performance.

\section{Atenção e tempo de reacção nos praticantes de karate Shotokan}

Resumo: 0 objectivo do presente estudo é analisar a capacidade de atenção e o tempo de reacção em praticantes de karate portugueses. Participaram 96 karatecas Shotokan federados e pertencentes ao Centro Português de Karate aos quais foram recolhidas medidas antropométricas (peso, altura, índice de massa corporal e percentagem de massa gorda), e foram avaliados quanto ao Tempo de Reacção Simple (TRS), Tempo de Reacção de Escolha (TRE), Tempo de Decisão (TD) e A tenção Distribuída (AD). Os dados foram analisados de acordo com a idade dos karatecas (15-19, 20-35 e mais de 35 anos), graduação ( $90-4^{\circ}$ kyu, $3^{\circ}-1^{\circ}$ kyu, Dan) e género (Masculino e Feminino). Os resultados demonstram que os karatecas masculinos, relativamente aos femininos apresentam características físicas significativamente diferentes quanto à altura, peso, percentagem de massa gorda e anos de prática. Quanto ao TRS todos os grupos tendem para que as respostas ocorram 300 ms depois de surgir o estímulo e sem diferenças significativas entre eles, mas relativamente ao TRE e ao TD, os karatecas com graduação de Dan e com mais de 35 anos apresentam tempos significativamente mais demorados que os restantes grupos. Por outro lado, os karatecas mais graduados (Dan) e mais com mais de 35 anos tendem a cometer menos erros nas suas escolhas. Relativamente ao género este não influência significativamente o tempo de reacção nos praticantes de karate Shotokan, mas parece que as mulheres avaliadas tendem a reagir mais rapidamente que os homens. Quanto à $A D$, podemos concluir que não existem diferenças significativas em função da graduação do atleta, nem em função do género. Encontrámos diferenças estatisticamente significativas em função da idade, ou seja, os atletas mais velhos foram aqueles que apresentaram níveis inferiores de atenção distribuída. Istos resultados parecem indicar que alguma coisa tem que ser modificado no processo de treino dos karatecas shotokan em Portugal.

Palavras-chave: Atenção, tempo de reacção, processamento de informação, karate, rendimento. 


\section{A tención y tiempo de reacción en practicantes de kárate Shotokan}

António Vences de Brito, Carlos Silva, luis Cid, Dora Ferreira, Ana Marques

IPS - Escola Superior de Desporto de Rio Maior (Portugal).

\section{Introducción}

Gracias al entrenamiento, se supone que los karatecas desarrollan un alto nivel de atención, lo que les permite detectar los estímulos más rápidamente y con mayor precisión, reduciendo así el tiempo de respuesta a los estímulos que crea el adversario.

El tiempo de reacción (TR) es una medida física relacionada con el rendimiento humano, pudiendo ser definido como el intervalo de tiempo que existe entre la presentación de un estímulo y el subsiguiente comportamiento de respuesta (Godinho, Mendes, Melo, \& Barreiros, 1999). En psicología es considerado un índice de velocidad de procesamiento de la información (Jensen, 2006), siendo generalmente aceptado que toda la información fluye, mediante impulsos nerviosos, en diversas fases, desde la captación/recepción de los estímulos hasta la ejecución motora, pasando por las estructuras de análisis y decisión (Massaro, 1989; W elford, 1980). Este tiempo de transformaciones, durante el cual la información "corre" por el sistema nervioso, fue denominado como cronometría mental (Posner \& Rogers, 1978). Actualmente, esta teoría es utilizada frecuentemente para el estudio de los mecanismos implicados en el procesamiento de la información, en especial en la toma de decisiones, una vez que traduce la rapidez con que el sujeto trata la información (Alves, 1995). A esto se denomina reacciometría (Alves, 1982), que entendida como la respuesta a un estímulo puede definirse como el método de medición de la velocidad de reacción, que se traduce en un movimiento voluntario de respuesta ante un estímulo externo. La forma más común para su medición es el TR.

También desde una perspectiva psicofisiológica, podemos decir que el TR representa el nivel de coordinación neuromuscular en el cual la estimulación visual, auditiva o táctil es descodificada por el cuerpo a través de diferentes procesos (físico-químicos y mecánicos), viajando así a través de las vías aferentes, llegando al cerebro como estímulos sensoriales. Seguidamente, la respuesta motora es transmitida por vías neuronales eferentes, pasando por la médula espinal y con- 
duciendo la respuesta a las unidades motoras que implementan la acción motora deseada (Der \& Deary, 2006).

Un indicador de este proceso es la velocidad del sujeto para ejecutar las operaciones mentales exigidas por la tarea, y así la velocidad de procesamiento de la información puede ser considerada como un índice de eficiencia, generándose respuestas comportamentales tan sencillas como apretar un botón, un movimiento de ojos, una respuesta oral u otro comportamiento observable.

El TR es más rápido cuando sólo existe una posible respuesta (tiempo de reacción simple, TRS) y es más lento cuando se introducen opciones de respuesta adicionales (tiempo de reacción de elección, TRE). De acuerdo con la Ley de Hick (1952), el TRE aumenta en proporción al logaritmo del número de alternativas de respuesta. Describe el tiempo que el sujeto tarda en tomar una decisión como resultado de las posibles opciones que se le presentan.

Empíricamente, el TR se asocia fuertemente con la edad. Es conocido y aceptado que el proceso de envejecimiento del individuo conduce a un incremento del TR, verificándose que los jóvenes adultos son los que reaccionan más rápidamente, perdiendo velocidad a lo largo de los años. De acuerdo con algunos autores (Alves, 1985; Godinho, 1985; Tavares, 1993; Chiviacowsky, \& Godinho, 1997), la capacidad de procesar la información, tanto en términos de cantidad como de velocidad, aumenta con la edad. Los resultados de la investigación revelan que las principales diferencias entre los niños y los adultos se sitúan a nivel de rapidez y calidad de la respuesta. A proximadamente a los 16 años de edad las diferencias entre los jóvenes y los adultos comienzan a atenuarse, obteniéndose una mayor convergencia en los tiempos obtenidos, y se verifica una mejora significativa en las áreas de reacciometría hasta la edad adulta (aproximadamente a los 25 años). Después, se produce una estabilización hasta aproximadamente los 45 años, período a partir del cual comienza a disminuir la velocidad de reacción. No obstante, la velocidad de reacción puede mejorarse con la práctica hasta un determinado nivel. En condiciones de fatiga o distracción la velocidad tiende a empeorar (Der \& Deary, 2006).

Según Christina \& Rose (1985), el TR puede ser dividido en dos fases distintas. En la primera fase, llamada pre-motora 0 de pre-tensión, se produce la descodificación del estímulo, hasta el registro de las primeras actividades eléctricas en el músculo, llevadas a cabo por el sistema nervioso periférico; en la fase motora 0 de tensión, las células musculares inician el proceso de contracción, hasta la realización de los primeros movimientos.

Otros investigadores consideran que el sistema nervioso central prepara la fase pre-motora, una vez determinado que las tareas del TRS están más relacionadas con factores mentales que con el sistema nervioso periférico.

Más allá de esto, existen múltiples factores que pueden influir en el TR del sujeto (esencialmente relacionados con sus características, las del contexto y las características de la tarea a realizar). Con todo, de acuerdo con Cid, Fonseca \& Alves (2003), la atención es uno de los factores cognitivos que tienen más influencia en la velocidad de reacción de los sujetos, siendo esa la razón por la cual ambos están íntimamente asociados. De hecho, según varios autores (A bernethy, 1993; Summers \& Ford, 1995), la capacidad limitada de procesar información está 
directamente ligada a los mecanismos de atención. Tal vez por esa razón podemos definir los procesos atencionales como una capacidad limitada de procesar información proveniente del entorno o del propio cuerpo (Godinho, 1985). Sea como fuere, los procesos atencionales pueden ser puestos en ecuación desde dos perspectivas. La primera como un proceso que permite dirigir y focalizar nuestros esfuerzos mentales hacia los estímulos relevantes en la realización de una tarea, en el sentido de seleccionar la información pertinente y bloquear 0 eliminar aquella que no lo es (Viana \& Cruz, 1996). De esta forma, según Cid (2006), parece evidente que la capacidad de atención tiene tres funciones principales: focalizar, seleccionar y bloquear información. La segunda, como un proceso capaz de modelar el procesamiento de la información, facilitando y/o inhibiendo el tratamiento de una determinada información, donde no solo comprende la capacidad de recepción de los estímulos y la percepción de la información que estos contienen, sino que también orienta los mecanismos de selección, decisión y ejecución en el procesamiento de la información (Godinho, 1985).

A lo largo de varias décadas, diversos estudios han demostrado la importancia que tienen estos dos factores (atención y tiempo de reacción) en el contexto deportivo, incluyendo los deportes de combate (Andrade, Belmonte, \& Viana, 2006; Mori, Ohtani, \& Imanaka, 2002; N eto, Bolander, Pacheco, \& Bir, 2009; 0 'Donovan, Cheung, Catley, McGregor, \& Strutton, 2006).

En lo que se refiere al TR en el ámbito deportivo, puede ser entendido como la habilidad para responder rápidamente, con una postura adecuada y controlada, a un estímulo que puede ser un movimiento, sonido o señal. A veces la reacción es más importante que la velocidad pura. En muchos deportes raramente se alcanza la velocidad máxima, y lo que se exige frecuentemente es una reacción explosiva, siendo esto característico del kárate. También sabemos que el TRS recibe una mayor influencia por las características genéticas que por el entrenamiento, siendo no obstante posible que los karatecas mejoren el TR a través de los procesos de entrenamiento en cuanto a su capacidad de ejecutar respuestas correctas (TRE). A modo de ejemplo:

a. En el kárate, en un movimiento defensivo de bloqueo de un ataque efectuado con los brazos 0 con las piernas, el karateca lee las señales visuales o motoras del adversario, lo que le permite adecuar su posicionamiento corporal y motor sobre la base de la recepción y
Momento de UnA COMPETICIÓN DEPORTIVA, EN EL QUE SE APRECIA LA REACCIÓN ANTE UN ATAQUE CON BLOQUEO Y CONTRAATAQUE SIMULTÁNEO.

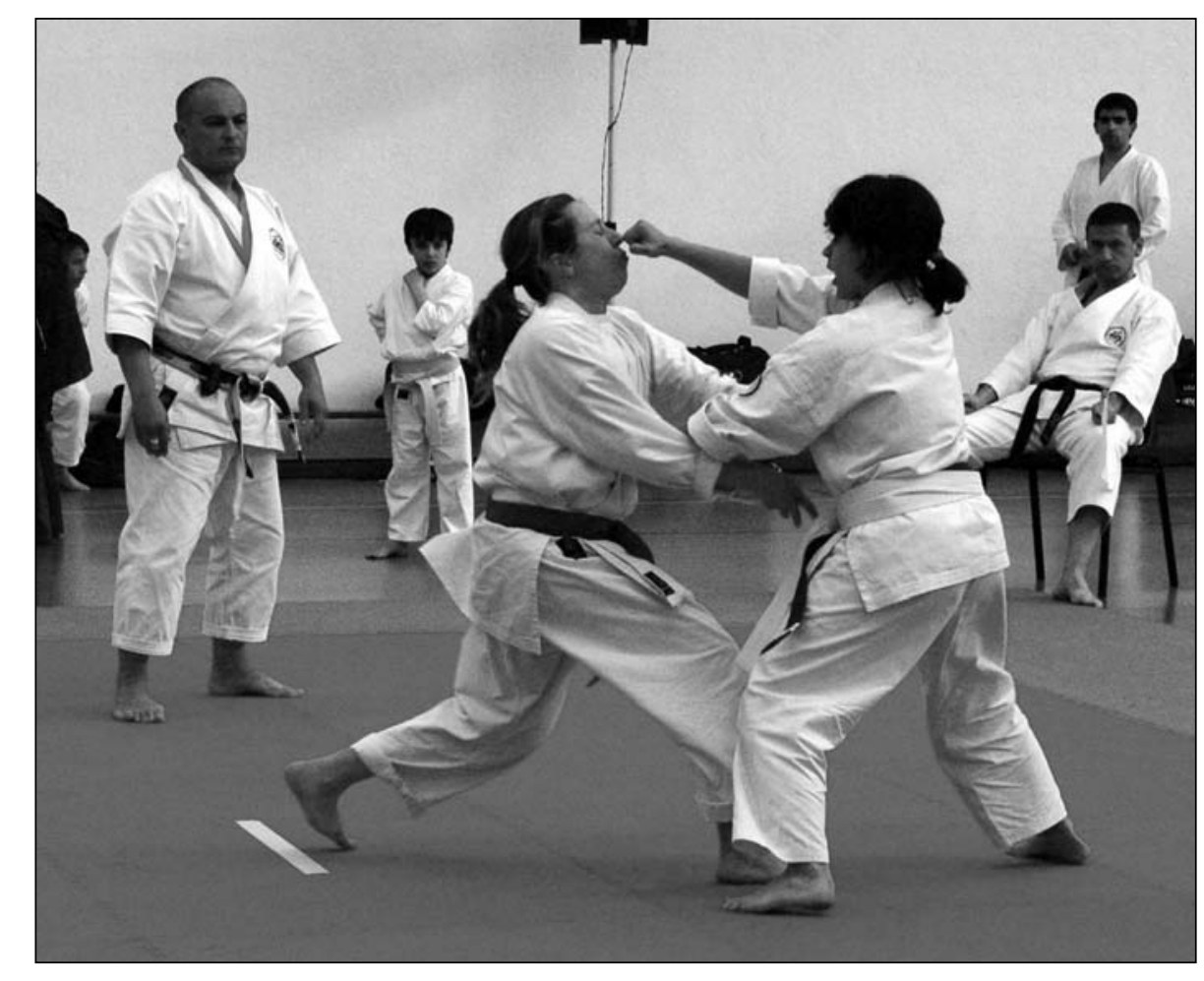


procesamiento de la información captada, y así reaccionar ante el ataque. Los karatecas con mayor éxito se anticipan, reaccionan de forma rápida y explosiva y de un modo controlado, como se ilustra en la imagen.

b. Una de las decisiones más importantes que el karateca tiene que tomar es si "avanza" o "no avanza", i.e., si ataca o no. Los karatecas con mayor éxito consiguen esperar más tiempo y reaccionar rápidamente. Esto permite al karateca leer los estímulos del adversario y decidir con mayor certeza si "avanza" o "no avanza".

Según Smith (1990), el TRE es el intervalo de tiempo más pequeño necesario para responder a un estímulo que se presenta como alternativa junto con otros estímulos. En kárate este tiempo es crucial para el rendimiento. Los métodos convencionales de entrenamiento del $T R$, basados en ejercicios específicos realizados entre dos individuos (W ichmann 1988, en Roosen, Compton, \& Szabo, 1999) son limitados porque al cabo de un tiempo comienza a existir previsibilidad en cuanto al inicio del movimiento del compañero (Roosen, Compton, \& Szabo, 1999). Este hecho va reduciendo el tiempo de reacción artificialmente, por anticipación, por lo que será muy eficiente en el entrenamiento; pero en la competición, donde el adversario es menos previsible, el TR pasa a ser mayor.

En el combate de kárate, la información a la que el karateca tiene que responder es la que tiene origen fundamentalmente en estímulos visuales, independientemente de si va a realizar un bloqueo 0 un ataque de brazos 0 piernas. La velocidad de la respuesta y del movimiento escogido para ejecutar son elementos críticos en el TR de respuesta, así como su correcta ejecución.

En el entrenamiento del kárate, la información más pertinente sobre el movimiento del adversario la dan los compañeros o el entrenador a través del feedback prescriptivo (N ewel \& McGinnis, 1985).

Por otro lado, en lo que se refiere al estudio de los procesos y mecanismos de atención en el deporte, siempre fue uno de los temas de mayor interés en el área de investigación de la psicología aplicada al contexto deportivo, puesto que su importancia es unánimemente reconocida, tanto en los procesos de entrenamiento para la competición, en función del alto rendimiento deportivo, como en los procesos de aprendizaje/formación deportiva (Viana \& Cruz, 1996; Cruz \& Gomes, 2001; Dosil, 2008). Es por este hecho (que el rendimiento deportivo está íntimamente ligado al acto de prestar atención a la tarea) que los entrenadores alertan constantemente a sus atletas sobre la importancia de mantener la atención y la concentración (A bernethy, Summers \& Ford, 1998). No obstante, a pesar de que siempre suelen ir de la mano, los conceptos de atención y concentración son diferentes, y se pueden definir del siguiente modo: la atención como una forma de interacción con el medio, en la que el sujeto establece contacto con los estímulos relevantes para la situación de ese momento presente; y la concentración como el mantenimiento de las condiciones atencionales a lo largo de un determinado período de tiempo (Dosil, 2008; Samulski, 2002; W einberg \& Gould, 2007).

En resumen, lo que es fundamental enfatizar tiene que ver con el hecho de que diversos autores evidencian, de una forma más o menos explícita, la importancia que tienen la atención y la velocidad de reacción en los deportes de 
combate (Cuadro, 2007; Gimeno \& Guedea, 2002; Pérez \& Morales, 2002). Considerando esta realidad, el objetivo de este estudio fue analizar el TR visual y la capacidad de distribución de la atención en practicantes de kárate en Portugal en función de su graduación (nivel de experiencia), edad y género.

\section{Metodología}

\section{Muestra}

En la realización del estudio participaron 96 karatecas Shotokan, masculinos y femeninos y con una edad igual o superior a 15 años, pertenecientes a la Federação Nacional de Karate - Portugal y representando al Centro Português de Karate. Todos ellos fueron previamente informados de los procedimientos del estudio y consintieron la utilización y divulgación de los resultados obtenidos.

Las características físicas de los karatecas de la muestra fueron: edad 31.1 \pm 14.1 años, peso $72.7 \pm 13.4 \mathrm{Kg}$, altura $171.2 \pm 8.1 \mathrm{~cm}$, índice de masa corporal (IMC) $24.7 \pm 3.8$ y porcentaje de masa grasa $(\% M G) 19.6 \pm 6.7$. En relación al tiempo de práctica los participantes entrenan $4.3 \pm 1.6$ horas por semana (HS) y acumulan $14.1 \pm 11$ años de práctica (AP).

\section{Instrumentos}

En la caracterización física de los karatecas, el peso, altura, IMC y \%MG se midieron con una balanza, una regla métrica y a través de bio-impedancia ( 0 mron BF300, Matsusaka, Japón). Para recoger los TR se utilizó el software "PRW in", desarrollado en el laboratorio de la Escola Superior de Desporto de Rio Maior - IPS por Sobreiro e Alves (2005). Este software, instalado en un ordenador portátil, permite recoger y evaluar el Tiempo de Reacción Simple (TRS - tiempo que transcurre entre la presentación de un estímulo visual, siempre con la misma localización, y la respuesta motora); Tiempo de Reacción de Elección (TRE - tiempo que transcurre entre la presentación de un estímulo visual, con cuatro localizaciones diferentes posibles, y la respuesta motora); y el Tiempo de Decisión (TD - diferencia entre TRS y TRE). La recogida de información se realizó en una sala donde los karatecas estaban aislados, y sin restricciones en cuanto a la posición de los brazos (véase imagen).
TEST DE TIEMPO DE REACCIÓN.

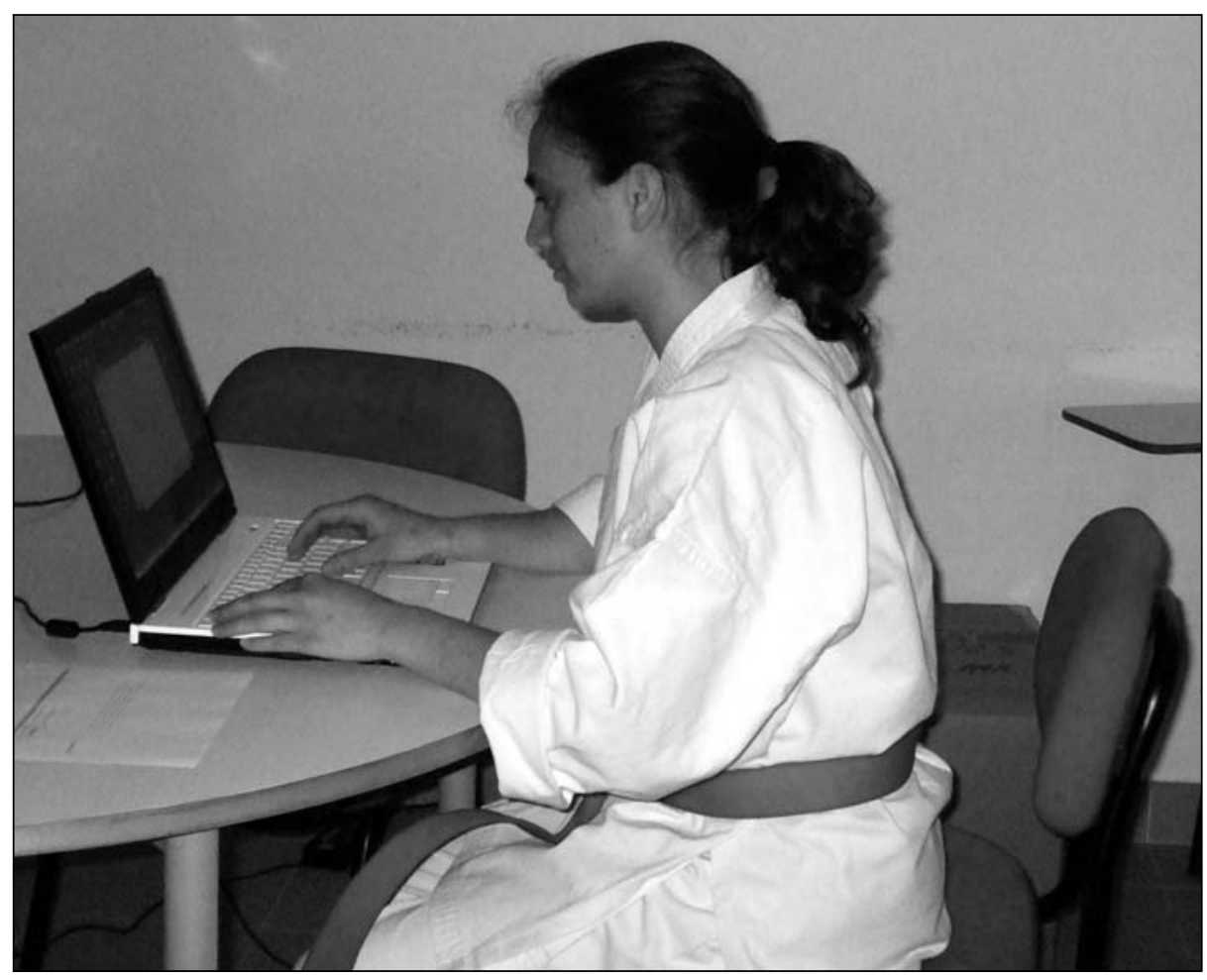




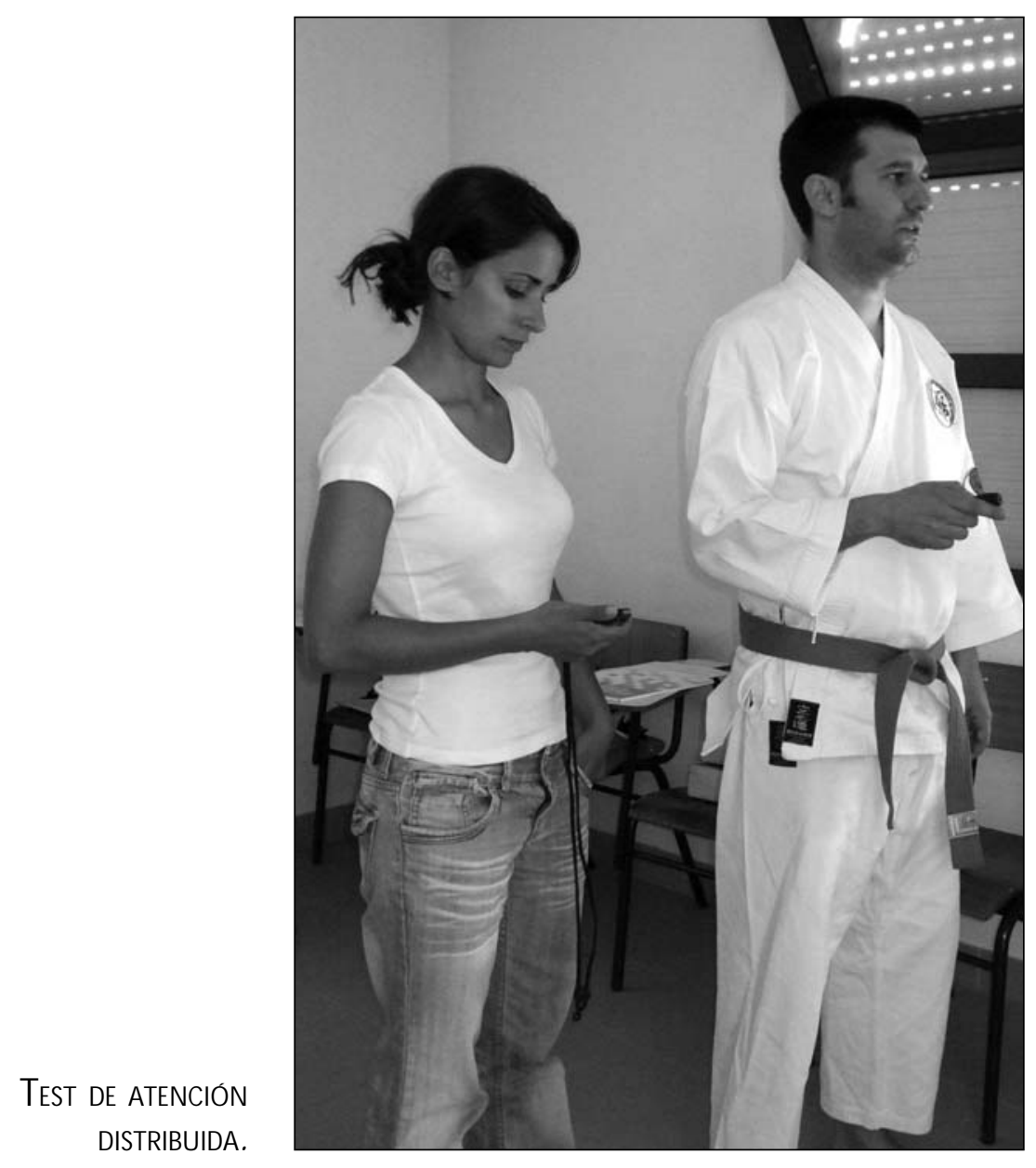

Para la recogida de datos relativos a la Atención Distribuida (AD) se utilizó el Cuadro de Schulte, compuesto por un cuadro metálico en el cual se disponen números del 1 al 38, distribuidos de forma aleatoria y con diversos tamaños. El cuadro fue colocado en una pared, con su centro a una altura de $1.60 \mathrm{~m}$, mientras que el sujeto se colocaba paralelamente al mismo a una distancia de $1.10 \mathrm{~m}$. En la realización de la tarea se pidió al sujeto que localizase e indicase de forma secuencial (apuntando con un puntero y verbalizando el número simultáneamente) todos los números del cuadro lo más rápidamente posible, como ilustra la imagen. El resultado final del test es el tiempo de ejecución en segundos.

Análisis de datos

Para el análisis de datos la muestra fue dividida en tres grupos distintos: según la edad (32 karatecas con edades entre los 15 y los 19 años, 27 karatecas entre 20 y 25 años, y 37 con más de 35 años), graduación (25 karatecas entre 9o y 40 kyu, 34 entre $3^{\text {er }}$ y $1^{\text {er }}$ kyu, y 37 con grado de dan), y género ( 75 hombres y 21 mujeres).

En la presentación de los resultados se utiliza una estadística descriptiva a través de las medias (M) y desviaciones estándar (SD). Para la verificación de la existencia de diferencias significativas entre los grupos se utilizó el análisis de la varianza (ANOVA) unidireccional y el T-test para muestras independientes. También se verificó la normalidad y homogeneidad de la muestra. En este tratamiento se consideró un grado de significación de $p \leq 0.05$. El procesamiento estadístico se realizó mediante el software SPSS 17.0.

\section{Resultados}

La Tabla 1 presenta los resultados obtenidos en cuanto a la caracterización física y tiempos de práctica total y semanal en los tres grupos establecidos. 
Tabla 1: caracterización física, AP y HS en los diferentes grupos.

\begin{tabular}{|c|c|c|c|c|c|c|c|}
\hline Graduación & $\begin{array}{l}\text { Edad } \\
\text { (años) } \\
\text { M / SD }\end{array}$ & $\begin{array}{c}\text { Altura } \\
\text { (cm) } \\
M / S D\end{array}$ & $\begin{array}{c}\text { Peso } \\
\text { ( kg) } \\
M / S D\end{array}$ & $\begin{array}{c}\text { IMC } \\
M / S D\end{array}$ & $\begin{array}{c}\% M G \\
M / S D\end{array}$ & $\begin{array}{c}\text { AP } \\
\text { (años) } \\
M / S D\end{array}$ & $\begin{array}{c}\text { HS } \\
\text { (horas) } \\
\text { M / SD }\end{array}$ \\
\hline 9o to $40 \mathrm{Kyu}$ & $26 \pm 10$ & $170 \pm 8$ & $71 * \pm 12$ & $25 * \pm 4$ & $21 \pm 6$ & $4 * \pm 3$ & $4 \pm 2$ \\
\hline $3^{\text {er to }} 1^{\text {er }} \mathrm{Kyu}$ & $21 \pm 9$ & $171 \pm 9$ & $68 \pm 14$ & $23 \pm 3$ & $17 \pm 7$ & $9 * \pm 4$ & $5 \pm 2$ \\
\hline Dan & $43 \pm 11$ & $173 \pm 7$ & $78 * \pm 12$ & $26 * \pm 4$ & $21 \pm 6$ & $24,9 * \pm 9$ & $4 \pm 1$ \\
\hline \multicolumn{8}{|l|}{ Edad } \\
\hline $15-19$ & $16 \pm 1$ & $170 \pm 8$ & $65^{*} \pm 12$ & $23 * \pm 3$ & $18 * \pm 7$ & $7 * \pm 3$ & $5 \pm 2$ \\
\hline $20-35$ & $26 \pm 7$ & $170 \pm 9$ & $70 \pm 13$ & $24 \pm 3$ & $18 \pm 7$ & $9 * \pm 6$ & $4 \pm 4$ \\
\hline+35 & $47 \pm 7$ & $173 \pm 7$ & $79 * \pm 12$ & $27 * \pm 3$ & $22 * \pm 5$ & $24 * \pm 12$ & $4 \pm 1$ \\
\hline \multicolumn{8}{|l|}{ Género } \\
\hline M & $33 \pm 15$ & $174 * \pm 6$ & $76 * \pm 12$ & $25 * \pm 4$ & $18 * \pm 7$ & $16 * \pm 11$ & $4 \pm 2$ \\
\hline $\mathrm{F}$ & $25 \pm 9$ & $162 \pm 8$ & $60 \pm 9$ & $23 \pm 4$ & $23 \pm 6$ & $8 \pm 7$ & $5 \pm 2$ \\
\hline
\end{tabular}

En cuanto al nivel de graduación, los karatecas con mayor grado tienen una edad mayor, un mayor peso, un mayor IMC y acumulan más años de práctica. El tiempo dedicado al entrenamiento por semana es semejante en todos los grupos. En función de la edad, se verifica que los karatecas mayores son significativamente más pesados, con mayor IMC y \%MG, y también son los que acumulan más años de práctica. En la distribución por género, se aprecia una tendencia a que los hombres sean más viejos que las mujeres, y son significativamente más altos, pesados, con mayor IMC, \%MG y con más años de práctica. Parece existir una tendencia a que las mujeres entrenen más horas a la semana que los hombres.

Seguidamente, la Tabla 2 presenta los resultados obtenidos por los diversos grupos en cuanto al tiempo de atención distribuida, tiempo de reacción simple, tiempo de reacción de elección, tiempo de decisión y errores cometidos.

Tabla 2: AD, TRS, TRE, TD y errores cometidos en los diferentes grupos.

\begin{tabular}{cccccc}
\hline Graduación & $\begin{array}{c}\text { AD (ms) } \\
\mathrm{M} \pm \mathrm{SD}\end{array}$ & $\begin{array}{c}\text { TRS (ms) } \\
\mathrm{M} \pm \mathrm{SD}\end{array}$ & $\begin{array}{c}\text { TRE }(\mathrm{ms}) \\
\mathrm{M} \pm \mathrm{SD}\end{array}$ & $\begin{array}{c}\text { TD (ms) } \\
\mathrm{M} \pm \mathrm{SD}\end{array}$ & $\begin{array}{c}\text { Errores } \\
\mathrm{M} \pm \mathrm{SD}\end{array}$ \\
\hline 90 to 40 $\mathrm{Kyu}$ & $139.8 \pm 27.0$ & $290 \pm 25$ & $426 \pm 45$ & $138 \pm 39$ & $4 \pm 2$ \\
30 to 10 $\mathrm{Kyu}$ & $140.2 \pm 32.9$ & $288 \pm 24$ & $429 \pm 50$ & $141 \pm 50$ & $3 \pm 3$ \\
Dan & $158.8 \pm 48.6$ & $295 \pm 32$ & $496 * \pm 80$ & $195 * \pm 67$ & $3 \pm 3$ \\
Edad & & & & & \\
\hline 15 - 19 & $146.6 \pm 34.1$ & $294 \pm 26$ & $423 \pm 50$ & $129 \pm 48$ & $4 \pm 3$ \\
20 - 35 & $136.4 \pm 35.6$ & $293 \pm 24$ & $443 \pm 51$ & $149 \pm 46$ & $3 \pm 3$ \\
+35 & $158.1 * \pm 45.1$ & $290 \pm 31$ & $501 * \pm 80$ & $205 * \pm 62$ & $3 \pm 2$ \\
Género & & & & & \\
\hline M & $149.1 \pm 42.5$ & $292 \pm 30$ & $458 \pm 77$ & $163 \pm 67$ & $3 \pm 2$ \\
F & $142.9 \pm 24.9$ & $288 \pm 20$ & $447 \pm 40$ & $159 \pm 35$ & $4 \pm 4$ \\
\hline
\end{tabular}

$* p \leq 0.05$ 
En relación a los resultados de la $A D$, se aprecia que no existen diferencias significativas en función de la graduación, a pesar de que los atletas con mayor experiencia (i.e., grado de dan) son aquellos que presentan peores resultados medios ( $M=158.8, S D=48.6)$, puesto que en la prueba realizada de atención distribuida los mejores resultados son los más bajos, mostrando que el atleta tardó menos en la realización del test. En lo que se refiere a la influencia de la edad, se verifica una tendencia semejante, puesto que los resultados indican que los atletas de mayor edad (i.e., más de 35 años) tienen una peor atención distribuida ( $M=158.1$, $\mathrm{SD}=45.1$ ) respecto a los más jóvenes ( $M=139.8, S D=27.0)$. No obstante, sólo se encuentran diferencias estadísticamente significativas con el grupo de atletas entre 25 y 35 años ( $M=136.4, S D=35.6)$. Por último, en lo que se refiere al género, no se encontraron diferencias estadísticamente significativas entre los grupos, a pesar de que en términos medios las mujeres presentan mejores resultados ( $M=149.1$, $\mathrm{SD}=42.5)$.

En relación al TR, se verifica que el TRS de los diferentes grupos sucede entre $288 \pm 24 \mathrm{~ms}$ y $295 \pm 32 \mathrm{~ms}$ tras el estímulo, sin que aparezcan diferencias significativas entre los grupos. Parece existir una tendencia a que las mujeres y los practicantes de mayor edad tengan un TRS menor. En el TRE las respuestas se producen entre los $423 \pm 50$ ms y los $501 \pm 80$ ms después del estímulo, verificándose que los karatecas con mayor graduación y con más de 35 años de edad son significativamente más lentos para reaccionar ante el estímulo visual presentado, lo que conduce a que el tiempo necesario para decidir efectuar una respuesta sea también significativamente mayor en los karatecas con dan y con más de 35 años. Por otro lado, se aprecia una tendencia a que el número de errores cometidos en la toma de decisión sea menor en estos dos grupos de karatecas así como en los hombres. A pesar de que en el análisis por género las mujeres tiendan a fallar más que los hombres, también tienden a responder más rápidamente y a tener un menor TD que los hombres.

\section{Discusión}

Como parte del kárate, de su aprendizaje y en asociación con los objetivos de su práctica, la necesidad de responder rápidamente, con eficacia y calidad a un estímulo externo que es inherente a la interacción existente con el adversario, exige la adecuación de las metodologías de entrenamiento al desarrollo de la capacidad de responder correcta y rápidamente a los estímulos que acontecen. De esta capacidad depende el éxito de la respuesta.

El tiempo de entrenamiento semanal y aquel que se va desarrollando a lo largo de los años, en función de su calidad, van a ser factores fundamentales para la mejora de los diversos gestos motores en articulación con el desarrollo y la adaptación de las capacidades neurosensitivas y coordinativas. Con todo, el envejecimiento progresivo y el género son factores que pueden constreñir las ejecuciones motoras, independientemente de la existencia de práctica deportiva.

Tal y como se identificó, los resultados relativos a las características físicas de los karatecas son diferentes entre los grupos formados por edad, en conjunción con el nivel técnico y también con el género. Esta diferencia no acontece en lo que se refiere al tiempo dedicado al entrenamiento durante la semana, donde 
todos los grupos parecen dedicar tiempos similares. Este hecho puede ser la razón que lleva a que los TRS encontrados no presenten diferencias significativas entre los grupos. Por otro lado, factores como los años de entrenamiento, el nivel de graduación 0 el género no se muestran como factores que afecten a los TRS de los karatecas Shotokan.

Según Roosen, Compton, \& Szabo (1999), el TRS no es muy importante para los practicantes del kárate. A pesar de que nuestros resultados pueden sustentar esta opinión, contrarían nuestras expectativas y los resultados de otros autores (Der \& Deary, 2006), que refieren que la edad (el envejecimiento) conduce a un aumento del TRS, y que este mejora con el entrenamiento. Así, podemos pensar que los sujetos de mayor edad de nuestra muestra se benefician de la influencia del entrenamiento porque sus TRS no son peores que los de los restantes grupos. De hecho, en un estudio realizado con atletas de taekwondo (Andrade, Belmonte, \& Viana, 2006), que tenía como uno de sus objetivos analizar las diferencias en la velocidad de reacción simple a estímulos visuales y auditivos entre dos grupos de atletas con diferentes edades (10-16 y 21-42 años), los resultados indicaron que los atletas de mayor edad fueron los más rápidos en las pruebas de reacciometría, lo que lleva a los autores a concluir que el TRS puede mejorarse con la práctica.

Sea como fuere, tal y como sugieren O'Donovan, Cheug, Catley, M cGregor \& Strutton (2006), que estudiaron una muestra compuesta por practicantes de diferentes artes marciales y un grupo control de no atletas, las mejoras en el TR de los atletas se debe sobre todo al tiempo de movimiento (ejecución motora), puesto que los autores no encontraron diferencias significativas entre atletas y no atletas en las tareas de reacciometría (TRS y TRE), pero descubrieron diferencias favorables a los practicantes de artes marciales en el tiempo de ejecución del movimiento.

Por otro lado, el TRS tiende a ser menor en las mujeres (W elford, 1980), aunque podemos encontrar resultados contradictorios en la literatura (Alves, 1985b). Los resultados del presente estudio concuerdan con los alcanzados por Tavares (1991), quien concluyó en su estudio que las mujeres obtuvieron un mejor TR en situaciones más complejas (TRE con cuatro estímulos), sin encontrar diferencias en el TRS.

La falta de diferenciación respecto al TRS entre los grupos puede deberse a la gran heterogeneidad de los practicantes que entrenan en los dojos de Portugal, teniendo que considerar también que el reducido número de horas de entrenamiento semanal limita la mejora de las capacidades de recepción/percepción, procesamiento y respuesta a los estímulos en tiempos cada vez más reducidos. Este problema tendrá más impacto en los karatecas con mayor graduación. Las clases habituales se forman generalmente con karatecas de todas las edades y con diversas graduaciones. Este proceso de organización de las clases, que se debe fundamentalmente a las limitaciones de utilización de los espacios físicos, condiciona el carácter corrector de la práctica, el aprendizaje y el desarrollo neuromotor y neurosensorial.

La respuesta rápida a un estímulo que surge en tiempos y con direcciones inesperadas es lo que constantemente se solicita al practicante de kárate. Esta solicitación permanente conduciría a que los karatecas con mayor nivel técnico y 
con más años de práctica tuviesen TRE y TD menores y cometiesen menos errores que los karatecas con menor graduación y edad. Contrariamente, lo que se verificó fue que los karatecas de mayor graduación y edad tenían TRE y TD mayores que los restantes karatecas, y con un número de errores semejante. Esto contraría las expectativas y los resultados de Mori, Ohtani \& Imanaka (2002), y va en la línea de lo señalado por Alves (1999) y Ferreira (1990), que refieren que la edad es un factor que conduce al aumento del TRE y TD. Por otro lado, en el caso de los karatecas de mayor graduación y más de 35 años de edad, este aumento del TRE puede estar influido también por la cualidad/carga del entrenamiento. Con todo, Roosen, Compton, \& Szabo (1999) recuerdan que en el kárate el TRE es crucial para el rendimiento, lo que implica que los karatecas portugueses de mayor graduación deberían modificar sus procesos de entrenamiento.

El género ha sido un factor sobre el que no se ha hallado un consenso en el estudio y análisis del TR; algunos autores señalan que las mujeres tienen TR mayores que los hombres (Alves, 1999; Landauer, Armstrong, \& Digwood, 1980; Neto, Bolander, Pacheco, \& Bir, 2009), y otros señalan lo contrario (W elford, 1980), estando en este caso la disminución del TR asociada a la práctica deportiva. En los karatecas analizados en nuestro estudio, a pesar de no haberse encontrado diferencias significativas entre géneros, no podemos concluir que el entrenamiento del kárate conduzca a una homogeneización del TR entre los hombres y las mujeres, ya que se verifica una tendencia a que el TR y el TD sean semejantes en ambos grupos.

En relación a la $A D$, los valores alcanzados en nuestro estudio indican que apenas existieron diferencias significativas entre los grupos en lo que se refiere a la edad. De hecho, los atletas mayores (mayores de 35 años) obtuvieron resultados significativamente mayores en el test de $A D$ en relación a uno de los grupos de atletas más jóvenes (entre 20 y 35 años). Estos resultados tienen lógica si se consideran las características de los grupos de estudio, puesto que como señalamos, la capacidad de los sujetos va mejorando significativamente desde la infancia a la madurez, llegando a su máximo exponente a partir de los 16 años hasta aproximadamente los 25. Después de un periodo de estabilización comienza a disminuir hasta aproximadamente los 45 años, y este es precisamente el escalón etario de la mayor parte de los atletas del grupo de más de 35 años.

Por otro lado, los resultados obtenidos parecen contradecir la literatura en lo que respecta a la influencia que tiene el nivel de maestría/graduación sobre los procesos atencionales. De hecho, no se encontraron diferencias estadísticamente significativas entre los grupos ( $F=2.584, p=0.081)$, y no deja de ser curioso que los atletas con un nivel técnico inferior obtengan mejores resultados medios que los atletas con más experiencia. Según algunos autores (Samulski, 1995; Viana \& Cruz, 1996), la experiencia y el nivel técnico más elevado proporcionan una mejor orientación de la atención a los estímulos que son más importantes para la tarea a realizar, y un atleta más experimentado consigue dominar una gran variedad de tareas, teniendo disponible una "energía atencional" (Samulski, 1995, p. 42) que pueda aplicar en nuevas situaciones, "ahorrando de esta manera los recursos atencionales" (Viana \& Cruz, 1996, p. 296). En la mayoría de los casos este hecho está asociado al desarrollo de mejores estrategias visuales y perceptivas especí- 
ficas de la modalidad (desarrolladas con el entrenamiento y con la participación en la competición), a través de las cuales los atletas se hacen más veloces y más selectivos en su mirada hacia la información que contienen los estímulos que les rodean (Godinho, 1985).

Por ello, tal vez la especificidad de la modalidad sea un factor determinante. $\mathrm{Si}$ tomamos en cuenta las características de la modalidad y las dimensiones del modelo de estilos atencionales de Niddefer (1976) (amplitud y dirección de la atención), podemos decir que el kárate Shotokan se caracteriza por ser una modalidad cuyas exigencias atencionales son más reducidas en términos de amplitud y externas en términos de dirección del foco atencional, por lo que el tipo de atención predominante en esta modalidad será la atención concentrada. Así, pensamos que la evaluación realizada a los atletas en cuanto a AD puede tener alguna influencia en los resultados por sus limitaciones en medir la atención concentrada. Este punto de vista parece ser reforzado por el estudio realizado por Fontani, Lodi, Feleci, Migliorini \& Corradeschi (2006), que al analizar las diferencias entre atletas más y menos experimentados en la modalidad de kárate, en relación a su capacidad de distribuir la atención, verificaron que los atletas más experimentados fueron aquellos que obtuvieron peores resultados y cometieron más errores.

\section{Conclusiones}

No podemos dejar de señalar y reforzar la importancia que tienen los procesos atencionales en el rendimiento deportivo, y que estos pueden marcar la diferencia entre el éxito y el fracaso de las acciones motoras de los atletas (Cruz \& Gomes, 2001; Dosil, 2008; Samulski, 2002; W einberg \& Gould, 2007; Viana \& Cruz, 1996). Como resultado de este trabajo, opinamos que las metodologías y estrategias de organización y de implementación de las sesiones de entrenamiento de kárate son factores esenciales para la modificación y mejora de los procesos atencionales y de los TR de los karatecas portugueses. El aumento del TRE y del TD en los karatecas con graduación de dan, y la semejanza del TRS entre todos los grupos son indicadores de que son necesarias modificaciones en el proceso de entrenamiento de los karatecas Shotokan en Portugal.

\section{REFERENCIAS}

Abernethy, B. (1993). Attention. In R. Singer, M. Murphey, \& K. Tennant (Eds.), Handbook of Research on Sport Psychology (pp. 127-170). New York: Macmillan Publishers.

Abernethy, B., Summers, J., \& Ford, S. (1998). Issues in the Measurement of Attention. En J. Duda (Ed.), Advances in Sport and Exercise Psychology M easurement (pp. 173-193). Morgantown: Fitness Information Technology Inc.

Alves, J. (1982). A reaciometria e as suas possibilidades. Ludens, 6(3), 34-38.

Alves, J. (1985). Relação entre o tempo de reacção simples, de escolha e de decisão e o tipo de desporto praticado (individual e colectivo). Provas Académicas. Lisboa: ISEF (documento no publicado). 
Alves, J. (1985b). 0 tempo de reacção. Provas Académicas. Lisboa: ISEF (documento no publicado).

Alves, J. (1995). Processamento da informação e inteligência. Lisboa: Edições $\mathrm{FMH}$.

Alves, J. (1999). Tempo de reacção e inteligência. En J. Alves, C. Serra \& N. Casanova (Eds.), A prendizagem M otora e Tomada de Decisão no Desporto (pp. 44-83). Guarda: Edições IPG.

Andrade, A., Belmonte, A., \& Viana, M. (2006). Tempo de reacção, flexibilidade e velocidade acíclica do membro inferior de atletas de Tae Kown Do. Lecturas: EFDeportes, Revista Digital, 96. Disponible en http://www.efdeportes.com/efd96/tkd.htm. [Consulta 25/11/2010].

Christina, R. \& Rose, D. (1985). Premotor and motor reaction time as a function of response complexity. Research Quarterly for Exercise and Sport, 56, 306-315.

Cid, L. (2006). A tenção e Performance Desportiva: Da Teoria à Prática. Horizonte, $125,8-14$.

Cruz, J. \& Gomes, R. (2001). A preparação mental e psicológica dos atletas e os factores psicológicos associados ao rendimento. Treino Desportivo, 16, $35-40$.

Cuadro, H. (2007). Critérios para la selección de talentos en deportes de combate. Lecturas: EFDeportes, Revista Digital, 104. Disponible en http://www.efdeportes.com/efd104/seleccion-de-talentos. htm. [Consulta 25/11/2010].

Der, G. \& Deary, I. (2006). Age and sex differences in reaction time in adulthood: Results from the United Kingdom health and lifestyle survey. Psychology and Aging, 21, 62-73.

Dosil, J. (2008). Psicología de la A ctividad Física y del Deporte. Madrid: M cGraw Hill.

Ferreira, V. (1990). Tempo de reacção simples, de escolha e de decisão. M otricidade Humana, 6(1-2), 117-131.

Fontani, G., Lodi, L., Felici, A., Migliorini, S., \& Corradeschi, F. (2006). Attention in Athletes of high and low experience engaged in different open skill sports. Perceptual and Motor Skills, 102, 791-805.

Gimeno, F. \& Guedea, J. (2002). Evaluación e intervención psicológica en judo. En J. Dosil (Ed.), El Psicólogo del Deporte. A sesoramiento e Intervención (pp. 301-324). Madrid: Editorial Sintesis.

Godinho, M. (1985). Processamento da informação, percepção visual e desenvolvimento motor. Ludens, 9(2), 15-17.

Godinho, M., Mendes, R., Melo, F., \& Barreiros, J. (1999). Controlo Motor e A prendizagem: Fundamentos e A plicações. Lisboa: FMH.

Hick, W. (1952). On the rate of gain of information. Quarterly Journal of Experimental Psychology, 4, 11-26.

Hyman, R. (1953). Stimulus information as a determinant of reaction time. Journal of Experimental Psychology, 45, 188-196.

Jensen, A. (2006). Clocking the mind: Mental chronometry and individual differences. Amsterdam: Elsevier. 
Landauer, A., Armstrong, S., \& Digwood, J. (1980). Sex difference in choice reaction time. British Journal of Psychology, 71, 551-555.

Massaro, D. (1989). Experimental psychology. An information processing approach. Orlando: Harcourt Brace Jovanovich Publishers.

Mori, S.; Ohtani, Y.; \& Imanaka, K. (2002). Reaction times and anticipatory skills of karate athletes. Human M ovement Sciences. 21(2), 213-230.

Neto, O., Bolander, R., Pacheco, M. \& Bir, C. (2009). Force, reaction time, and precision of Kun Fu strikes. Perceptual and Motor Skills, 109(1), 295303.

Newell, K. \& McGinnis, P. (1985). Kinematic information feedback for skilled performance. Human Learning, 4, 39-56.

Nideffer, R. (1976). Test of Attentional and Interpersonal Style. Journal of Personality and Social Psychology, 34, 394-404.

O'Donovan, O., Cheung, J., Catley, M., McGregor, A., \& Strutton, P. (2006). An investigation of leg and trunk strength and reaction times of hard-style martial arts practitioners. Journal of Sports Science and Medicine, 5, $5-12$.

Pérez, 0.\& Morales, F. (2002). La táctica como instrumento de mejoras de la velocidad de reacción en los deportes de combate. Lecturas: EFD eportes, Revista Digital, 53. Disponible en http://www.efdeportes.com/efd53/tacti.htm. [Consulta 25/11/2010].

Posner, M. \& Rogers, M. (1978). Chronometric analysis of abstraction and recognition. In W. Estes (Ed.), Handbook of Learning and Cognition Processes (pp. 143-188). Hillsdale, N ew Jersey: Erlbaum.

Roosen, A., Compton, G. \& Szabo, A. (1999). A device to measure choice reaction time in karate. Sports Engineering, 2(1), 49-54.

Samulski, D. (1995). Psicologia do esporte: Teoria e aplicação prática. Belo Horizonte: Imprensa Universitária/UFM G.

Samulski, D. (2002). Psicologia do Esporte. S.Paulo: Editora Manole.

Schmidt, R. (1990). M otor Learning and Performance: From principles to practice. Champaign, Illinois: Human Kinetics.

Singer, R. (1986). El aprendizaje de las acciones motrices en el deporte. Barcelona: Editorial Hispano Europea.

Sobreiro, P. \& Alves, J. (2005). PRW in: Software para a mensuração do tempo de reacção. En A. Vitorino, A. Ramires, C. Borrego, C. Silva, J. Martins, J. Alves, L. Cid, M. Gouveia, P. Almeida, \& P. Sobreiro (Eds.), Actas do II Congresso Internacional de Psicologia do Desporto. Rio Maior: Edições ESDRM.

Summers, J. \& Ford, S. (1995). Attention in sport. En T. Morris e J. Summers (Eds.), Sport Psychology: Theory, applications and issues (pp. 63-89). Chichester: W iley.

Tavares, F. (1991). Estudo da relação entre os tempos de reaç̧ão simples, de escolha e de decisão, e o tipo de desporto praticado nos dois sexos. En J. Bento $\& A$. Marques (Eds.), As ciências do desporto e a prática desportiva, 2, 55-63. Porto: Edições FCDEF. 
Tavares, F. (1993). A capacidade de decisão táctica no jogador de basquetebol: Estudo comparativo dos processos perceptivo-cognitivos em atletas seniores e cadetes. Tesis Doctoral. Porto: FCDEF (documento no publicado).

Viana, M. \& Cruz, J. (1996). Atenção e concentração na competição desportiva. En J. Cruz (Ed.), Manual de Psicologia do Desporto (pp. 287-304). Braga, Sistemas Humanos.

Weinberg, R. \& Gould, D. (2007). Foundations of Sport and Exercise Psychology. Champaign Illinois: Human Kinetics.

W elford, A. (1980). Choice reaction time: Basic concepts. En A. W elford (Ed.), Reaction Times (pp. 73-128). New York: Academic Press

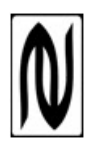

